\title{
UTILIZACIÓN DE HÁBITAT POR REPTILES EN ESTADOS SUCESIONALES DE SELVAS TROPICALES DE CAMPECHE, MÉXICO
}

\author{
R. René Calderón-ManduJano', Carlos Galindo-Leal ${ }^{2}$, \\ y J. Rogelio CEDEÑo-VÁzQuEz ${ }^{1}$ \\ ${ }^{1}$ El Colegio de la Frontera Sur-Unidad Chetumal, Av. Centenario Km 5.5, \\ Chetumal QUINTANA ROO. CP 779014, AP 424. \\ 2 Fondo Mundial para la Naturaleza (WWF-México), Av. México 51, Col. Hipódromo, \\ México, D.F. 06100, MÉXICO \\ rcalderon@ecosur.mx
}

RESUMEN

Este estudio documenta la utilización de hábitat por reptiles en selvas mediana y baja, con tres estados sucesionales: menor de 10 años, entre 10 y 25 años y selvas maduras (> 30 años). La investigación se llevó a cabo entre abril de 1997 y enero de 1998 en la Reserva de la Biosfera Calakmul, Campeche, México. Los muestreos se realizaron a lo largo de un año para incluir las tres estaciones climáticas predominantes en la región: nortes, secas y lluvias. Utilizamos métodos complementarios de muestreo: búsqueda directa en transectos y fuera de ellos, y muestreo con trampas, para evitar sesgos. La información recabada se analizó mediante técnicas multivariadas y de ordenación, para identificar tendencias o afinidades de las especies hacia cierto estado de la vegetación. Se encontraron 10 especies en mayor abundancia y representación en al menos 5 de los 6 tratamientos, que fueron utilizadas para comparar las diferencias entre los sitios conservados de los perturbados.

\begin{abstract}
This study documents patterns of habitat use by reptiles in low and medium height tropical forest in three successional stages: less than 10 years old, between 10 and 25 years old, and mature ( $>30$ years old) forests. The investigation took place between April 1997 and January 1998 in the Calakmul Biosphere Reserve, Campeche, Mexico. Sampling took place throughout one year period to include the three predominant seasons in the region: dry, wet and nortes. To avoid sampling biases, we used complementary methods: direct search in transects and outside transects, and trapping. We used multivariate and ordination techniques to identify trends in habitat use. Once that patterns of habitat use were identified, we used analysis of variance to test differences in abundance. We identified 10 species with high abundance, inhabiting at least in five of the six treatments, which were used to compare differences between preserved and disturbed sites.
\end{abstract}


Calderón Mandujano et al.: Utilización de hábitat por reptiles en selvas tropicales

\section{INTRODUCCIÓN}

La fragmentación del hábitat es un proceso que afecta de manera diferencial la diversidad biológica, ya sea facilitando o condicionando el desarrollo de los organismos por espacio, competencia, depredación, etc. (Baldwin et al. 2004). Los cambios generados a partir del desarrollo de las actividades humanas se han reflejado en la reducción y deterioro del hábitat natural, lo cual a su vez ha impactado de manera sensible las poblaciones silvestres en todo el mundo (Schlaepfer et al. 2005). Para la evaluación de la calidad del hábitat es relativamente nuevo el uso de vertebrados y aún existen discrepancias debido a la falta trabajos confiables (Landres et al. 1988). La mayor parte de esos trabajos se han centrado en anfibios (Lips et al. 2001), mamíferos (ej. murciélagos; Gorresen \& Willing 2004) y otros grupos taxonómicos como aves e invertebrados (Bierregaard et al. 1992). Recientemente, también los reptiles se han estudiado con estos fines (Smith \& Royce 2001, Urbina-Cardona et al. 2003, 2005, en prensa).

La herpetofauna tiene funciones muy importantes en los ecosistemas como depredadores y presas, y debido a su biología pueden ser considerados como indicadores de la integridad ambiental (Tuberville et al. 2005). Los anfibios y reptiles, por su condición ectotérmica, son más susceptibles a cambios en el entorno que afectan la temperatura, fuerza y dirección del viento, humedad, etc. (Schlaepfer \& Gavin 2001). Anteriormente se habían utilizado las serpientes como indicadores de contaminación en áreas de cultivo ya que éstas asimilan los agroquímicos (Bauerle et al. 1975). En las Antillas Menores, se observó que algunas poblaciones de lagartijas han disminuido como resultado de la perturbación (cambios en la cobertura vegetal, reducción del hábitat, contaminantes, entre otros) en sus hábitats (Roughgarden \& Pacala 1989). Recientemente, en México se evaluó el cambio en la diversidad de especies de anfibios y reptiles a lo largo de un gradiente de perturbación que incluyó potreros, bordes e interiores de la selva; además de correlacionar algunas variables ambientales y del hábitat con las abundancias de las especies (Urbina-Cardona, en prensa), encontrando una estrecha relación entre la composición y abundancia de éstos grupos y las variables ambientales en cada gradiente.

Algunos investigadores consideran que algunas especies de reptiles, y específicamente algunas lagartijas de los géneros Ameiva, Anolis y Sceloporus, pueden ser útiles para identificar posibles cambios ambientales (Muñoz et al. 1996, Hager 1998, Hofer et al. 2000). Estos géneros presentan gran diversidad de especies, amplia distribución en el continente americano y alta diversidad de especialización hacia determinados ambientes (Duellman 1963).

El objetivo de este estudio fue identificar los gremios o ensambles de reptiles que están más relacionados con la edad de recuperación del hábitat. Evaluamos las abundancias de los reptiles en dos tipos de vegetación con tres estados de madurez, analizando la abundancia de las especies en lugar de la sola presencia de las mismas. 


\section{MATERIALES Y MÉTODOS}

El trabajo de campo se realizó de abril de 1997 a enero de 1998 en la Reserva de la Biosfera Calakmul, en el sureste del estado de Campeche, México $\left(19^{\circ} 15^{\prime}, 17^{\circ} 45^{\prime}\right.$ N; $90^{\circ} 10^{\prime}, 89^{\circ} 15^{\prime}$ O) (Fig. 1).

La vegetación presente en el área comprende ocho tipos de asociaciones vegetales (Miranda \& Hernández 1963, INEGI 1990, Martínez \& Galindo-Leal 2002). Sin embargo, muestreamos únicamente los dos tipos de vegetación más extensos y que presentaban las características de sucesionales necesarias para el estudio:

a) La selva mediana (subperennifolia) se caracteriza porque sus árboles pierden entre el 25 y $50 \%$ de sus hojas en lo más acentuado de la época seca; alcanza una altura del estrato arbóreo de entre 15 y $25 \mathrm{~m}$; se desarrolla en climas cálidos y subhúmedos, con una temperatura promedio anual mayor a $20^{\circ} \mathrm{C} \mathrm{y}$ una precipitación anual promedio superior a $1200 \mathrm{~mm}$. Las especies más comunes en este tipo de vegetación son, entre otras, el zapote (Manilakara zapota), el ramón (Brosimum alicastrum), el sak paj (Byrsonima bucidaefolia), la huaya (Talisia olivaeformis), el tzalam (Lysiloma latisilicua), el cha'ka (Bursera simaruba) y el chechem (Metopium brownei).

b) La selva baja se caracteriza por tener suelos profundos, cuyo drenaje puede variar desde rápido hasta muy deficiente, provocando que durante la época lluviosa se encuentren completamente inundados. Sus árboles alcanzan entre ocho y 15 metros de altura y pierden del 50 al 75\% de sus hojas en la época seca. Se desarrolla en condiciones de temperatura y humedad similares a las selvas medianas pero dependiendo de la humedad pueden ser subcaducifolias o subperennifolias. Las especies más comunes varían, pero entre sus elementos se puede encontrar chechem (M. brownei), cha'ka (B. simaruba), tsisilche' (Gynopodyum floribundum), huaya ( $T$. olivaeformis) y palo de tinte (Hematoxylon campechianum).

Se denomina vegetación secundaria o "acahuales" a la vegetación que se desarrolla como resultado de la alteración de su estado original (Islebe 1998). En el caso de la península de Yucatán se sabe que existen muy pocos lugares que no hayan sido alterados debido al aprovechamiento de los bosques que se ha hecho durante siglos (Martínez \& Galindo-Leal 2002). Para este trabajo, los estados conservados fueron sitios cuya vegetación no ha sido alterada en un tiempo mayor a 30 años y los estados perturbados fueron sitios que son resultado de las actividades agrícolas (rozatumba y quema), que involucran la pérdida de la vegetación y que además presentan un grado de regeneración menor y mayor a 10 años. 


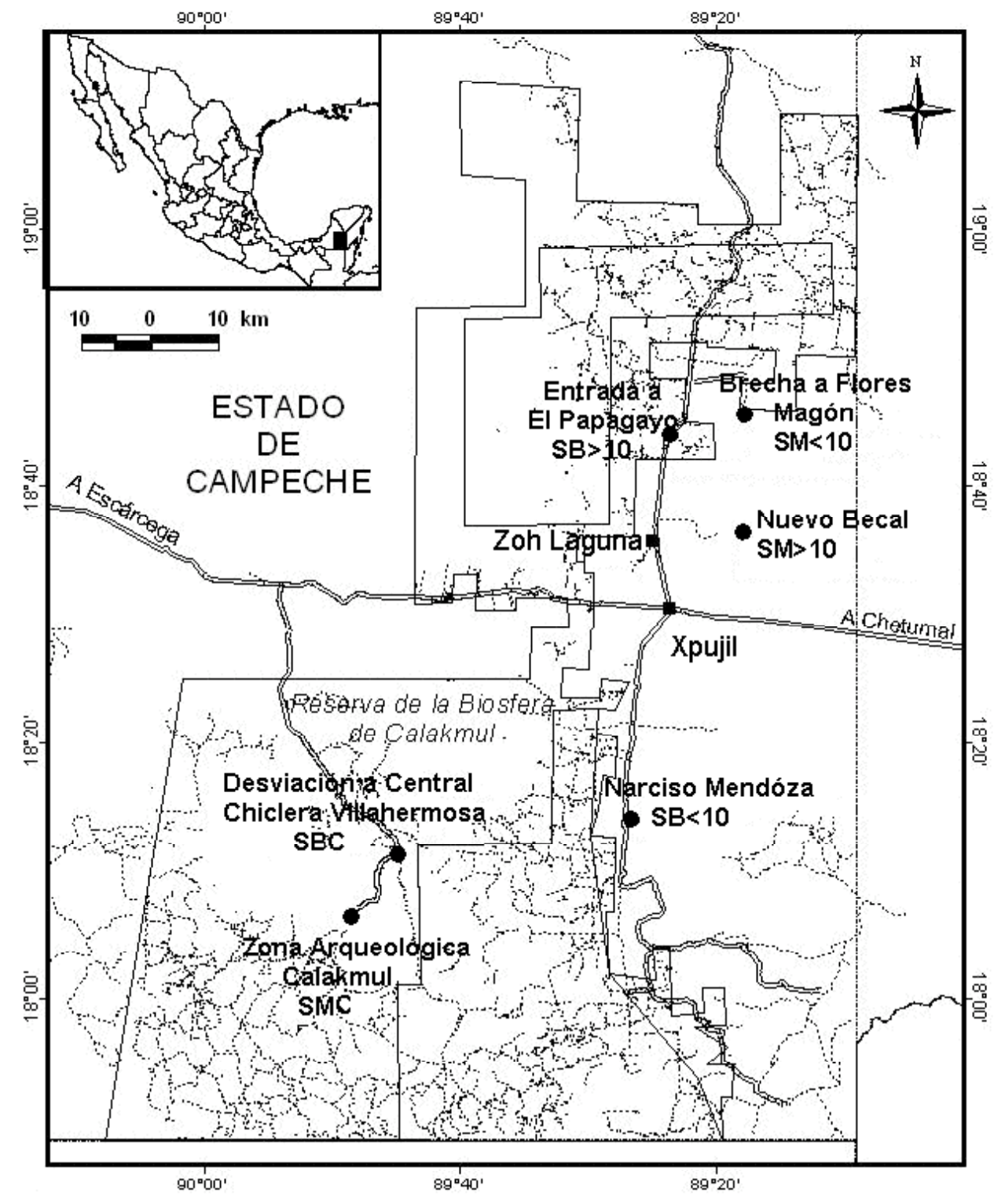

Figura 1

Área de estudio y tipos de vegetación presentes. $(\mathrm{SB}<10)$ acahual de selva baja $(<10$ años); $(\mathrm{SB}>10)$ acahual de selva baja ( $>10$ años); (SBC) selva baja conservada; $(\mathrm{SM}<10)$ acahual de selva mediana $(<10$ años); (SM>10) acahual de selva mediana ( $>10$ años); (SMC) selva mediana conservada. 
En ambos tipos de selva la composición del estrato arbóreo cambia drásticamente en los diferentes estados sucesionales. En el estado menor de 10 años dominan las especies arbustivas, palmas y helechos, y el estrato arbóreo es de baja densidad. Conforme va madurando la vegetación hacia la etapa de más de 10 años de recuperación y en el estado maduro, el estrato herbáceo pierde densidad, los arbustos son de mayor tamaño y las palmas tienden a ser más dominantes, así mismo el estrato arbóreo se encuentra más denso.

Se efectuaron ocho campañas de muestreo con duración de 12 días cada una. Se ubicaron seis zonas de trabajo en tres estados sucesionales de selva mediana y selva baja (selva madura (con más de 30 años de recuperación) y estados mayores de 10 y menores de 10 años). En cada campaña se muestrearon tres zonas durante cuatro días y sus respectivas noches (Cuadro 1). Cada zona contaba con tres réplicas (transectos) y se muestrearon de manera simultánea por espacio de cuatro días. Los muestreos se realizaron mediante dos métodos: a) muestreo con trampas y b) búsqueda dirigida.

\section{Cuadro 1}

Número de transectos y esfuerzo de muestreo por tipo y condición de la vegetación en la Reserva de la Biosfera Calakmul: $(\mathrm{ASB}<10)$ acahual de selva baja $(<10$ años $)$; $(\mathrm{ASB}>10)$ acahual de selva baja $(>10$ años); $(\mathrm{SBC})$ selva baja conservada; $(\mathrm{ASM}<10)$ acahual de selva mediana ( $<10$ años); (ASM $>10)$ acahual de selva mediana ( $>10$ años); (SMC) selva mediana conservada.

\begin{tabular}{|c|c|c|c|c|c|c|c|}
\hline Tipo de vegetación & $\mathrm{ASB}<10$ & $\mathrm{ASB}>10$ & SBC & $\mathrm{ASM}<10$ & $\mathrm{ASM}>10$ & SMC & Total \\
\hline Núm. Transectos & 3 & 3 & 3 & 3 & 3 & 3 & 18 \\
\hline Campañas/tipo de & 4 & 4 & 4 & 4 & 4 & 4 & 24 \\
\hline \multicolumn{8}{|l|}{ vegetación } \\
\hline Días/Campaña & 4 & 4 & 4 & 4 & 4 & 4 & 128 \\
\hline Horas/Día & 4 & 4 & 4 & 4 & 4 & 4 & \\
\hline Total Horas / & 192 & 192 & 192 & 192 & 192 & 192 & 1152 \\
\hline \multicolumn{8}{|l|}{ Hombre } \\
\hline No. Cercos/ & 9 & 9 & 9 & 9 & 9 & 9 & 54 \\
\hline \multicolumn{8}{|l|}{ Transecto } \\
\hline Tiempo/Trampa & 1152 & 1152 & 1152 & 1152 & 1152 & 1152 & 6912 \\
\hline
\end{tabular}


a) Muestreo con trampas: se ubicaron tres transectos de $500 \mathrm{~m}$ en cada uno de los sitios seleccionados, iniciándose en un camino y dirigidos perpendicularmente al interior del hábitat muestreado. Cada uno de los transectos contó con tres cercos de desvío a 100, 300 y $500 \mathrm{~m}$. Los cercos consisten en láminas galvanizadas de $15 \mathrm{~m}$ de longitud y $60 \mathrm{~cm}$ de altura, colocadas en el suelo. A cada uno de los lados de las láminas se colocaron tres trampas (seis por lámina) de malla mosquitera de aluminio, para que los organismos se introdujeran y quedasen atrapados (Fig. 2). Los transectos se muestrearon tres días en cada sitio revisando $2 \mathrm{~h}$ durante el día (09:00 - 11:00) y $2 \mathrm{~h}$ durante la noche (20:00 $-22: 00)$. Los organismos que se observaron en el transecto en ese lapso fueron registrados.

b) Búsqueda dirigida: Se realizó búsqueda dirigida en los lugares más propicios a la localización de anfibios y reptiles. Esta búsqueda se llevó a cabo en horas que no interfirieran con la revisión de los transectos y que son más susceptibles de actividad de estos organismos, tanto para las especies diurnas como para las nocturnas (de 11:00 a 13:00 y de 22:00 a 24:00 h).

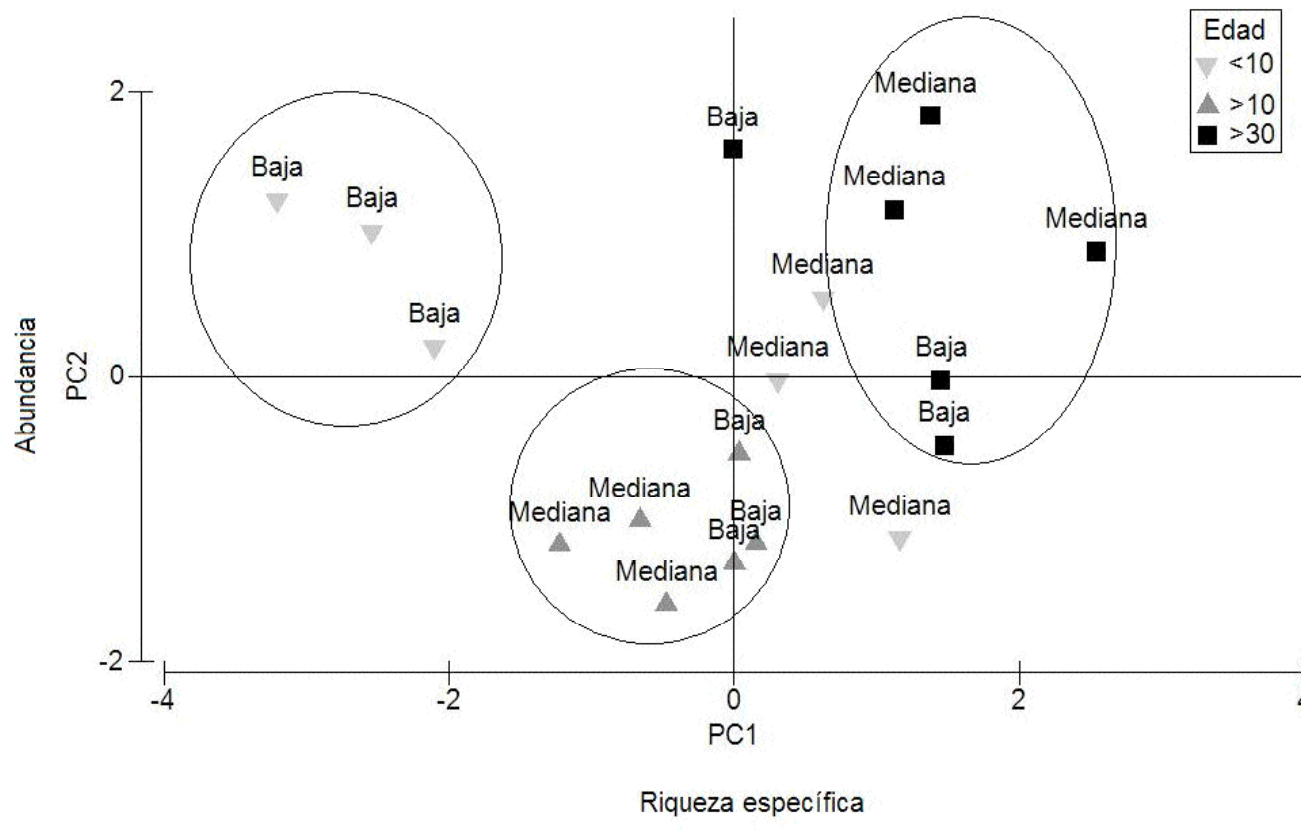

Figura 2

Análisis de componentes principales (PCA), con la ordenación de los tipos de vegetación considerando la composición de los ensambles de especies. Tipo de selva: baja y mediana; edad de recuperación: $<10$ años, $>10$ años, $>30$ años. Los grupos afines se indican mediante los círculos. 


\begin{abstract}
ANÁLISIS DE DATOS
Para los análisis estadísticos se utilizó únicamente la información generada de manera sistemática en los transectos. La información obtenida fuera de los transectos se utilizó para obtener una lista completa de los ejemplares del área.

Con el propósito de normalizar los datos, éstos se transformaron a $\log (\mathrm{X}+1)$. Posteriormente se realizó un análisis de componentes principales (PCA) para separar las especies por grupos afines de acuerdo al grado de perturbación del hábitat. Se calculó el valor de importancia de cada una de las variables mediante un análisis SIMPER (porcentajes de similitud y contribución de especies), calculado de dos vías cruzado entre la vegetación y la edad de recuperación. Posteriormente se realizó un agrupamiento (Cluster) de los tipos de vegetación y la edad de recuperación a partir de una matriz de similitud usando el índice de de Bray-Curtis, para identificar cuales se parecen más en función de la abundancia y riqueza de las especies. Se realizó un análisis multidimensional no métrico (MDS) con el mismo fin (Herrando-Pérez 2000). Se aplicó un análisis de dos vías anidado de tipo ANOSIM, que funciona de manera similar a un análisis de varianza pero de manera múltiple. Los valores que genera el ANOSIM se expresan en función del grado de disimilitud entre los diferentes tratamientos (en este caso la vegetación y los estados de conservación). Todos los análisis se realizaron mediante el programa Primer Ver. 6 (Licencia AP5200).
\end{abstract}

\title{
RESULTADOS
}

Después de las 8 campañas de muestreo se acumuló un total de 1152 horas hombre $(\mathrm{H} / \mathrm{H})$ y 6912 horas trampa $(\mathrm{H} / \mathrm{T})$ en los 18 transectos que se muestrearon sistemáticamente. Otra proporción igual de esfuerzo $(\mathrm{H} / \mathrm{H})$, se acumuló en los muestreos fuera de transectos, sin embargo; no fueron equitativas a los tipos de vegetación elegidos debido a que se incluyeron otras asociaciones vegetales y cuerpos de agua presentes en el área. Se registró un total de 61 especies de reptiles (Anexo 1), únicamente las 38 encontradas en los transectos fueron utilizadas en los análisis.

Los estados de vegetación que presentaron el mayor número de registros fueron los estados con más de 30 años de recuperación de la selva mediana y de la selva baja. Se aprecian dos tendencias en cuanto a las abundancias de las especies: aquellas que aumentan en los estados maduros y aquellas que por el contrario, son más abundantes en los estados sucesionales (Cuadro 2).

El análisis mediante PCA describe una separación entre la composición de especies abundantes de reptiles en los tipos de vegetación, en los que la selva mayor de 30años (baja y mediana) y los estados sucesionales se sitúan opuestos en el plano. De acuerdo a lo observado en el Cuadro 2, en los ejes uno y dos (PCA1 y PCA2), el primero está 
Calderón Mandujano et al.: Utilización de hábitat por reptiles en selvas tropicales

\section{Cuadro 2}

Especies de reptiles, su abundancia, riqueza específica y valor de importancia en los dos ejes principales (PCA) en cada condición de la vegetación en la Reserva de la Biosfera Calakmul: $(\mathrm{SB}<10)$ acahual de selva baja $(<10$ años); $(\mathrm{SB}>10)$ acahual de selva baja $(>10$ años); (SBC) selva baja conservada; $(\mathrm{SM}<10)$ acahual de selva mediana $(<10$ años); $(\mathrm{SM}>10)$ acahual de selva mediana ( $>10$ años); (SMC) selva mediana conservada.

\begin{tabular}{|c|c|c|c|c|c|c|c|c|}
\hline ESPECIE & $\mathrm{SB}<10$ & $\mathrm{SB}>10$ & SBC & $\mathrm{SM}<10$ & $\mathrm{SM}>\mathbf{1 0}$ & SMC & PCA1 & PCA2 \\
\hline Anolis lemurinus & 1 & 2 & 12 & 25 & 0 & 27 & -0.525 & -0.337 \\
\hline Ameiva undulata & 27 & 10 & 48 & 5 & 5 & 24 & 0.074 & -0.52 \\
\hline Anolis rodriguezii & 7 & 15 & 29 & 13 & 6 & 23 & -0.308 & 0.02 \\
\hline Coleonyx elegans & 3 & 1 & 5 & 3 & 1 & 15 & -0.166 & -0.486 \\
\hline Anolis tropidonotus & 0 & 5 & 11 & 3 & 6 & 9 & -0.281 & 0.291 \\
\hline Ninia sebae & 0 & 0 & 1 & 1 & 0 & 8 & -0.197 & -0.227 \\
\hline Corytophanes cristatus & 0 & 3 & 1 & 0 & 0 & 3 & -0.082 & -0.019 \\
\hline Dipsas brevifacies & 0 & 0 & 1 & 0 & 0 & 3 & -0.083 & -0.178 \\
\hline Corytophanes hernandezii & 0 & 0 & 1 & 0 & 0 & 2 & -0.038 & -0.149 \\
\hline Leptodeira frenata & 1 & 0 & 0 & 0 & 0 & 2 & -0.034 & -0.053 \\
\hline Scinella cherriei & 0 & 0 & 0 & 1 & 0 & 2 & -0.055 & -0.118 \\
\hline Ficimia publia & 0 & 2 & 0 & 0 & 0 & 2 & -0.069 & -0.021 \\
\hline Micrurus diastema & 0 & 0 & 2 & 1 & 1 & 1 & -0.084 & 0.068 \\
\hline Thecadactylus rapicauda & 0 & 0 & 1 & 0 & 0 & 1 & -0.046 & -0.038 \\
\hline Pseudoelaphe flavirufa & 0 & 0 & 1 & 0 & 0 & 1 & -0.071 & -0.013 \\
\hline Leptodeira septentrionalis & 0 & 0 & 0 & 0 & 1 & 1 & 0.002 & 0 \\
\hline Pliocercus elapoides & 0 & 0 & 0 & 0 & 0 & 1 & -0.02 & -0.039 \\
\hline Imantodes tenuissimus & 0 & 0 & 0 & 0 & 0 & 1 & -0.024 & -0.061 \\
\hline
\end{tabular}




\begin{tabular}{|c|c|c|c|c|c|c|c|c|}
\hline ESPECIE & $\mathrm{SB}<10$ & $\mathrm{SB}>10$ & SBC & $\mathrm{SM}<10$ & $\mathrm{SM}>\mathbf{1 0}$ & SMC & PCA1 & PCA2 \\
\hline Leptophis mexicanus & 0 & 0 & 0 & 0 & 1 & 1 & -0.036 & 0.024 \\
\hline Bothrops asper & 0 & 0 & 0 & 2 & 0 & 1 & -0.041 & -0.078 \\
\hline Tropidodipsas sartorii & 0 & 1 & 0 & 0 & 0 & 1 & -0.045 & 0.014 \\
\hline Drymobius margaritiferus & 0 & 2 & 4 & 1 & 1 & 0 & -0.075 & 0.155 \\
\hline Sibon nebulata & 1 & 0 & 1 & 0 & 0 & 0 & 0.057 & -0.094 \\
\hline Mastigodryas melanolomus & 1 & 0 & 1 & 0 & 0 & 0 & 0.011 & -0.006 \\
\hline Laemanctus serratus & 0 & 0 & 1 & 0 & 0 & 0 & 0 & -0.053 \\
\hline Imantodes cenchoa & 0 & 2 & 1 & 0 & 0 & 0 & -0.003 & 0.005 \\
\hline Symphimus mayae & 0 & 0 & 1 & 1 & 0 & 0 & -0.011 & -0.071 \\
\hline Rhinoclemmys areolata & 0 & 1 & 1 & 0 & 0 & 0 & -0.026 & 0.059 \\
\hline Ctenosaura alfredschmidti & 0 & 0 & 1 & 0 & 0 & 0 & -0.026 & 0.001 \\
\hline Xenodon rabdocephalus & 0 & 0 & 1 & 0 & 0 & 0 & -0.026 & 0.001 \\
\hline Sceloporus chrysostictus & 32 & 0 & 0 & 0 & 3 & 0 & 0.514 & -0.218 \\
\hline Basiliscus vittatus & 23 & 0 & 0 & 2 & 1 & 0 & 0.397 & -0.21 \\
\hline Coniophanes imperialis & 0 & 2 & 0 & 0 & 2 & 0 & 0.033 & 0.102 \\
\hline Kinosternon scorpioides & 0 & 0 & 0 & 0 & 1 & 0 & 0.022 & 0.039 \\
\hline Oxybelis fulgidus & 0 & 0 & 0 & 0 & 1 & 0 & 0.008 & 0.053 \\
\hline Anolis pentaprion & 0 & 1 & 0 & 0 & 0 & 0 & -0.001 & 0.018 \\
\hline Tropidodipsas fasciata & 0 & 1 & 0 & 0 & 0 & 0 & -0.003 & 0.04 \\
\hline Mabuya unimarginata & 0 & 0 & 0 & 1 & 0 & 0 & -0.005 & 0.001 \\
\hline Total general & 96 & 48 & 125 & 59 & 30 & 129 & & \\
\hline No. de especies & 9 & 14 & 21 & 13 & 13 & 21 & & \\
\hline
\end{tabular}


determinado por la riqueza especifica de cada sitio y el segundo por la abundancia. El gráfico resultante muestra la formación de tres grupos donde destacan, de manera más compacta, los acahuales de selva baja menores a 10 años de recuperación. Los estados con mayor recuperación se sitúan en el plano opuesto, de acuerdo al primer eje (PCA1), y a un nivel similar por parte del segundo eje (PCA2). En el caso de los sitios con recuperación media (mayor de 10 años), a pesar de que su diversidad es más alta, la abundancia es la más baja del resto de los sitios, por lo que ocupan el extremo inferior del segundo eje. En este caso es importante notar los valores particulares de cada especie en cada eje (PCA1 y 2 , Cuadro 2), ya que a priori son las que determinan la mayor parte de los análisis.

En el análisis SIMPER los valores de importancia, expresados en porcentaje, nos indican cuales especies hacen que la ordenación de los tratamientos se de en uno u otro plano de los gráficos. Se puede apreciar que la abundancia de 10 especies (Ameiva undulata, Anolis lemurinus, Anolis rodriguezii, Anolis tropidonotus, Basiliscus vittatus, Coleonyx elegans, Sceloporus chrysostictus, Drymobius margaritifens, Ninia sebae y Micnurus diastema) domina el ordenamiento y determinan la formación de los grupos en los gráficos (Cuadro 3). La importancia de estas diez especies recae en el hecho de que al hacer las comparaciones entre los sitios, sus abundancias varían de un sitio a otro pero siempre están presentes en al menos dos de los tratamientos para cada tipo de vegetación. Por el contrario, al hacer la comparación en el caso de las especies que sólo se encuentran de manera única, los valores son extremos.

En el agrupamiento (Cluster) resultante, los tipos de vegetación se van separando en función de la edad de recuperación más que por el tipo de la misma. Se aprecia un grupo compacto y cercano donde los transectos con vegetación tanto de selva baja como mediana con más de 30 años de recuperación se diferencian del resto. También, el grupo de selva baja con menos de 10 años se diferencia del resto de los acahuales de manera más clara; igualmente el resto de los transectos se agrupan de manera compacta, aunque a un nivel de similitud más bajo al punto de corte (Fig. 3). El punto de corte se estableció de acuerdo al promedio de similitud que arrojó cada grupo en el análisis SIMPER (55.885) (Cuadro 3).

En el análisis multidimensional (MDS), el gráfico resultante presenta un orden muy similar al que se presentan tanto en el PCA como en el agrupamiento (Cluster), quedando relativamente cercanos en la ordenación los estados maduros de ambas selvas, los acahuales de selva baja con menos de 10 años en otro grupo separado y el resto con una ordenación más dispersa. El valor de estrés obtenido (0.16), indica que el ajuste de la ordenación, es decir de los grupos que se forman entre las diferentes muestras o tratamientos, es bueno (Herrando-Pérez 2000) (Fig. 4).

Finalmente, el análisis de varianza aplicado (ANOSIM) nos muestra que la composición y abundancia de las especies encontradas en ambos tipos de vegetación (selva baja y mediana) no es significativa ( $100 \%$ de similitud); sin embargo, en el caso de la edad de recuperación la diferencia es alta (0.1\%) (Cuadro 4). Esto se puede 
Porcentaje de grupo

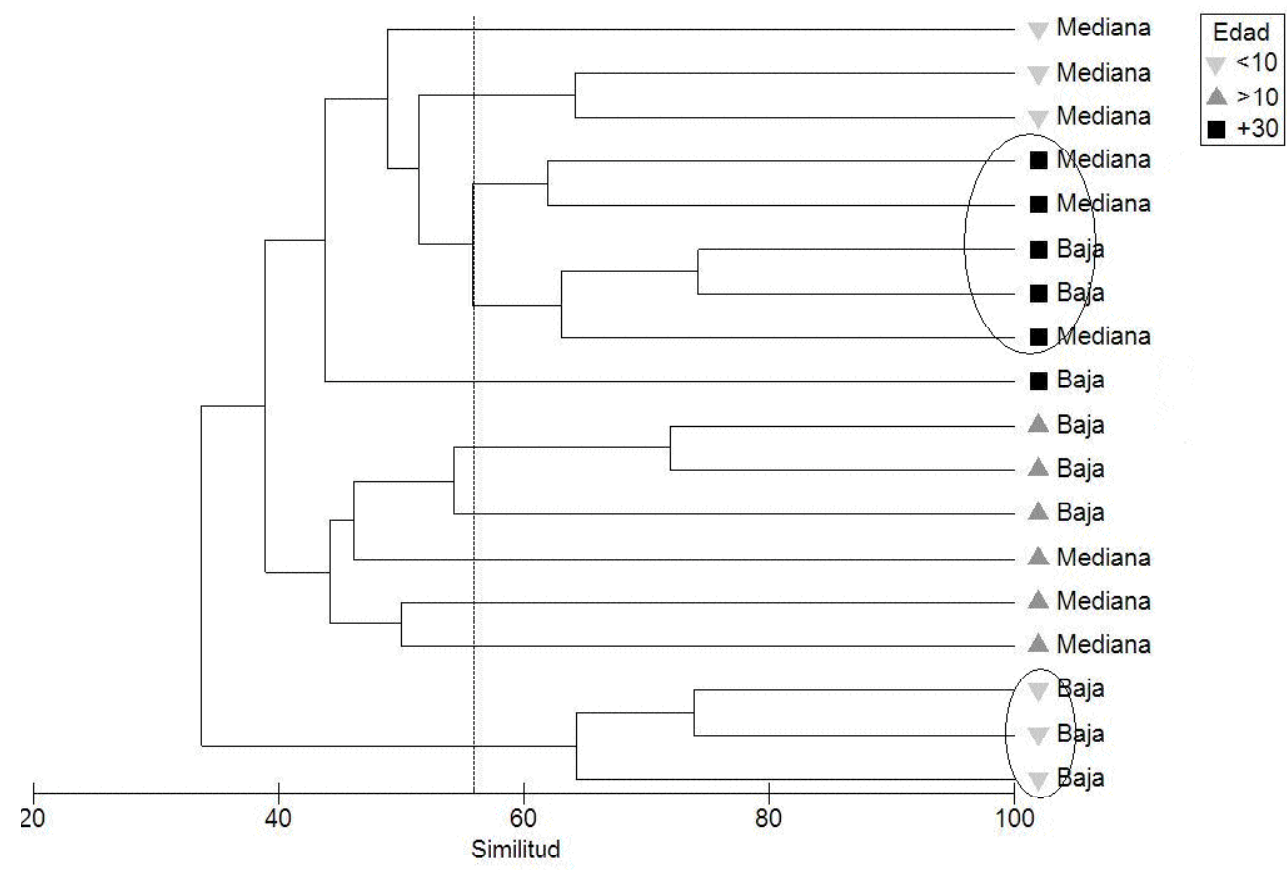

Figura 3

Similitud de la composición del ensamble de reptiles en los tipos de vegetación (Bray-Curtis). Tipo de selva: baja y mediana; edad de recuperación: $<10$ años, $>10$ años, $>30$. Los grupos afines se indican mediante los círculos.

interpretar como el hecho de que la composición y abundancia de los reptiles en selva baja y mediana, está fuertemente influenciado por el tiempo de recuperación de un sitio, más que por la estructura misma de estos dos tipos de vegetación. Las diferencias entre grupos son mayores en acahuales mayores de 10 años de recuperación con el resto de los tratamientos, principalmente con los estados de menos de 10 años de recuperación (Cuadro 3).

Los resultados observados muestran ensambles de especies cuyas abundancias cambian con el grado de recuperación del hábitat. Por un lado la selva baja presento un ensamble compuesto por $A$. undulata, A. rodriguezii, S. chrysostictus, B. vittatus, A. tropidonotus, A. lemurinus, C. elegans, Imantodes cenchoa, Corytophanes cristatus, D. margaritiferus y M. diastema. Para la selva mediana, el ensamble de especies se constituyó por A. rodriguezii, A. lemurinus, A. undulata, C. elegans, A. 
Calderón Mandujano et al.: Utilización de hábitat por reptiles en selvas tropicales

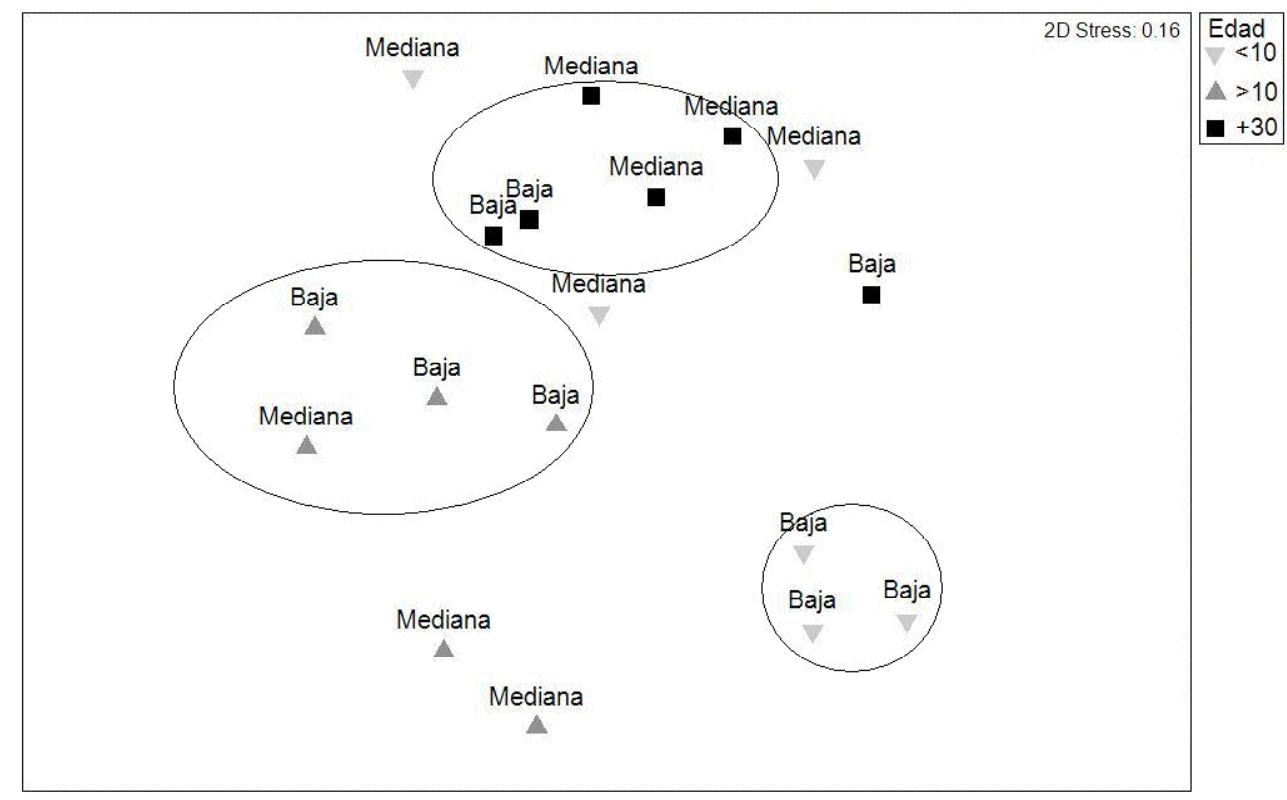

Figura 4

Análisis multidimensional no métrico (MDS), realizado a partir de una matriz de similitud (BrayCurtis). Tipo de selva: baja y mediana; Edad de recuperación: $<10$ años, $>10$ años, $>30$. Los grupos afines se indican mediante los círculos.

tropidonotus, Ninia sebae, Coniophanes imperiales, B. vittatus, Bothrops asper, $C$. cristatus, Scinella cherriei, Dipsas brevifacies y Ficimia publia. Las diferencias en los ensambles en estados con menor tiempo de recuperación recayó en la ausencia o aumento de la abundancia de algunas de estas especies (Cuadros 2 y 3 ).

\section{DISCUSIÓN}

Los ensambles de especies encontrados muestran de forma muy general, como se encuentran constituidos los gremios de reptiles de las selvas bajas y medianas de Calakmul. El análisis se encuentra dominado por diez especies, cuyas abundancias son las que determinan en gran medida las ordenaciones y los resultados de las pruebas estadísticas. La diferenciación de los sitos y su agrupamiento, en su mayoría, responden a especies conspicuamente abundantes, como es el caso de los sitios en selva baja menor de 10 años donde B. vittatus abunda, o el caso de Ameiva y los Anolis en los estados de mayor recuperación. 
Acta Zool. Mex. (n.s.) 24(1) (2008)

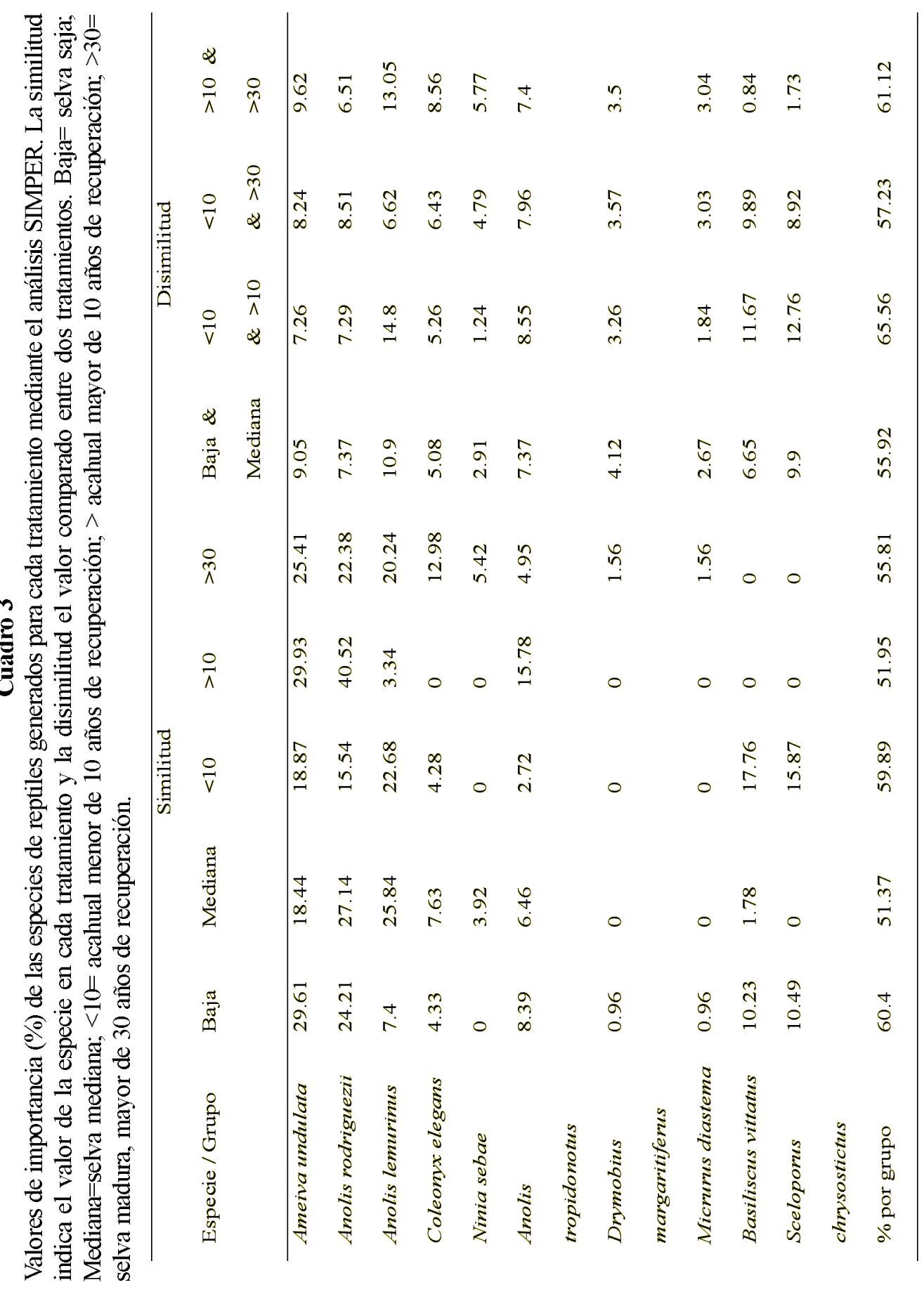


Calderón Mandujano et al.: Utilización de hábitat por reptiles en selvas tropicales

\section{Cuadro 4}

Análisis de varianza múltiple (ANOSIM) entre los estados de la vegetación y la edad de recuperación de la misma. El valor de significancia está dado en el porcentaje de similitud entre los grupos.

\begin{tabular}{lcc}
\hline Prueba para diferencias entre grupos & EDAD & VEGETACIÓN \\
\hline Muestra estadística (R Global) & 0.704 & -0.296 \\
Nivel de significancia de la muestra & $0.10 \%$ & $100 \%$ \\
\hline
\end{tabular}

Entre las diez especies más abundantes, tres fueron serpientes y siete lagartijas. Tres de éstas últimas fueron del género Anolis. Tres especies son de hábitos nocturnos (C. elegans, N. sebae, M. diastema), el resto son diurnas. Seis de las especies son de hábitos terrestres (A. undulata, C. elegans, S. chrysostictus, D. margaritiferus, $N$. sebae y $M$. diastema), las del género Anolis y B. vittatus son arborícolas.

Los estados de selva madura son los que registraron el mayor número de especies y de ejemplares de cada especie, los estados con más de 10 años de recuperación aunque cuentan con un mayor número de especies son los que presentan un menor número de registros (Cuadro 2). Este puede ser un efecto de colonización por parte de algunas especies generalistas en los estados menos conservados, ya que existe un mayor número de registros de pocas especies, mientras aumenta la edad de recuperación, aumenta el número de especies y la competencia entre éstas (Begon et al. 2006).

La diferencia en cuanto al número de registros entre estados maduros y estados sucesionales tempranos es clara. Algunos autores comentan que cuando la vegetación se encuentra en recuperación algunas poblaciones tienden a recolonizar nuevamente el área (Hanski 1994). Otros autores mencionan que en los estados secundarios, la presencia de caminos y veredas constituyen pequeños ecotonos, que a su vez concentran una elevada cantidad de organismos por presentar características apropiadas para su movilización (Andrews 1990). Contrariamente a estas teorías y a la teoría de la perturbación media (Connel 1978), los resultados encontrados mediante el muestreo sistemático muestran que los estados menos perturbados fueron los que registraron mayor número de especies.

Al igual que en el caso de otros grupos como los murciélagos (Gorresen \& Willing 2004), donde la abundancia de algunas especies es mayor en áreas maduras que en áreas de moderada perturbación y áreas perturbadas, las tres especies del género Anolis presentan la misma tendencia. En ambos tipos de selva se aprecia el aumento de sus abundancias en los estados con mayor tiempo de recuperación. En 
este caso la especie podría considerarse como indicadora de algún estado de la vegetación, pero habría que tener referencias sobre su comportamiento en otros tipos de vegetación y si éste se mantiene a lo largo de su distribución geográfica.

De acuerdo con Hirth (1963) Ameiva y Basiliscus son géneros que compiten en cuanto a sus hábitos alimentarios y territoriales, sin embargo, en este trabajo no se considera esto como cierto ya que ambas especies presentan hábitos y habitats diferentes, por lo que es dudoso que la distribución y abundancia de cada especie pueda estar condicionada por la otra. Ameiva undulata fue la especie más abundante en general y al igual que en otras especies, su abundancia fue mayor en los estados más recuperados. Basiliscus vittatus fue conspicuamente abundante en selva baja menor de 10 años, y sus valores seguramente son los que separan estos sitios en los gráficos de ordenación.

Coleonyx elegans y Scelopurus chrysostictus son especies que prefieren diferentes estados de recuperación de los dos tipos de vegetación en el área de estudio. Coleonyx elegans es más abundante en el estado primario de la selva mediana y baja. A pesar de ello, existe la duda sobre su respuesta a los hábitats en recuperación, ya que en los estados de recuperación media su abundancia fue inferior que en los estados de menor recuperación. Por otra parte, S. chrysostictus es una especie que se encuentra mejor representada en los estados mas perturbados. Por sus hábitos se encuentra con mayor facilidad en áreas abiertas (Lee 1980, 1996, Campbell 1998), incluyendo otros tipos de vegetación como sabanas y pedregales los cuales no fueron considerados en el análisis, pero que son importantes de destacar dada la naturaleza del estudio.

Las serpientes (Drymobius margaritifens, Ninia sebae, Micrurus diastema), presentan mayores registros en algún tipo y estado de recuperación de la vegetación. Esto puede estar más relacionado con sus hábitos más que con la condición del hábitat. Ninia sebae es terrestre, de hábitos nocturnos y semifosoriales, que se alimenta de invertebrados pequeños y prefiere lugares con humedad y abundante materia orgánica (Lee 1996, Calderón-Mandujano et al. 2005). M. diastema tiene hábitos similares, sólo que incluye a $N$. sebae en su dieta, lo cual podría estar relacionado con la distribución de ambas (Lee 1996). En cambio, D. margaritiferus es un especie diurna y se alimenta principalmente de anfibios (Lee 1996), los cuales tienden a ser más abundantes en vegetación con estratos altos que conservan mejor la humedad.

El valor de significancia obtenido mediante el análisis de varianza, indica que efectivamente hay diferencias entre la diversidad y la abundancia de los organismos en los diferentes estados de la vegetación. Las diferencias mayores se dan entre las edades de recuperación de la vegetación y no entre los tipos de vegetación. Esto puede ser consecuencia de las condiciones en las que se encuentran los tipos de vegetación en el área, a manera de mosaicos o parches muy cercanos entre sí, por lo que el intercambio de especies es muy factible. 
Calderón Mandujano et al.: Utilización de hábitat por reptiles en selvas tropicales

A manera de conclusión, se puede decir que la edad de recuperación del hábitat influye en el desarrollo de las especies y esto se refleja en el aumento o decremento de sus poblaciones. Al analizar los ensambles de especies de reptiles en la selva baja y mediana se observa que son muy similares. Las diferencias entre los gremios de reptiles presentes en los diferentes tiempos de recuperación del hábitat es mayor que las que existen entre los dos tipos de selva: baja y mediana. De manera más específica destacan los géneros Anolis, Coleonyx y Ameiva como grupos importantes de estudio con un alto potencial como especies indicadoras del estado de madurez de la selva y por lo tanto de su estado de conservación. Ameiva undulata es la especie más abundante en la selva baja y mediana de Calakmul, y sus abundancias se acentúan en estados con mayor tiempo de recuperación. Sceloporus chrysostictus es más abundante en donde la vegetación es más abierta, por lo que, dependiendo de las condiciones del área, se puede utilizar para evaluar el efecto de borde y el grado de perturbación como efecto de la alteración antropogénicas sobre la vegetación. Coleonyx elegans es una especie con mayor abundancia en la selva mediana madura lo que puede ser consecuencia de alguna característica específica de este tipo de vegetación. En todos los casos es necesario estudiar la biología de las especies para determinar los factores que determinan su predilección de hábitat. Debido a sus hábitos y a su amplia distribución es prematuro considerarlas como especies indicadoras hasta conocer más sobre su biología y ecología.

\section{AGRADECIMIENTOS}

Agradecemos a la Comisión Nacional para el Conocimiento y Uso de la Biodiversidad (CONABIO) y a El Colegio de la Frontera Sur (ECOSUR), el haber financiado los proyectos J112 (Inventario y monitoreo de Anfibios y Mariposas de la Reserva de la Biosfera de Calakmul, Campeche) y Q049 (Inventario y monitoreo de Anfibios, Reptiles y Mariposas de la Reserva de la Biosfera de Calakmul, Campeche) y a PRONATURA, A.C. Capítulo Península de Yucatán, que aportó fondos adicionales. A Oscar Flores-Villela y Gunther Köhler por verificar la determinación taxonómica de los especimenes de referencia depositados en la Colección Herpetológica (ECO-CH-H) del Museo de Zoología de ECOSUR. Al personal de guardia del INAH del sitio arqueológico Calakmul, por el apoyo y facilidades otorgados durante los muestreos. A ECOSUR, que facilitó el mobiliario necesario. Agradecemos también a Carmen Pozo que fue co-responsable de los proyectos antes mencionados. Oscar Flores-Villela y David González-Solís revisaron y aportaron comentarios valiosos al escrito final. Janneth A. Padilla Saldívar elaboró el mapa del área de estudio. A todo el personal de campo: Margarito y Alejandro Tuz, Santiago Uc, Pablo Beutelspacher, Aixchel Maya, Enrique Escobedo y José Luis Salinas, por el apoyo y la experiencia compartida. A los revisores asignados por la revista y al editor de la misma, cuyos comentarios aclararon las ideas para dar la forma final al trabajo. 


\section{ANEXO}

\begin{tabular}{|c|c|c|c|}
\hline FAMILIA & ESPECIE & FAMILIA & ESPECIE \\
\hline $\begin{array}{l}\text { Boidae } \\
\text { Colubridae }\end{array}$ & $\begin{array}{l}\text { Boa constrictor } \\
\text { Coniophanes imperialis } \\
\text { Coniophanes schmidti } \\
\text { Dipsas brevifacies } \\
\text { Drymarchon melanurus } \\
\text { Drymobius margaritiferus } \\
\text { Ficimia publia } \\
\text { Imantodes cenchoa } \\
\text { Imantodes tenuissimus } \\
\text { Lampropeltis triangulum } \\
\text { Leptodeira frenata } \\
\text { Leptodeira septentrionalis } \\
\text { Leptophis ahaetulla } \\
\text { Leptophis mexicanus } \\
\text { Mastigodryas melanolomus } \\
\text { Ninia sebae } \\
\text { Oxybelis aeneus } \\
\text { Oxybelis fulgidus } \\
\text { Pliocercus elapoides } \\
\text { Pseudoelaphe flavirufa } \\
\text { Pseustes poecilonotus } \\
\text { Sibon nebulata } \\
\text { Spilotes pullatus } \\
\text { Symphimus mayae } \\
\text { Tantillita lintonii } \\
\text { Thamnophis marcianus } \\
\text { Tropidodipsas fasciata } \\
\text { Tropidodipsas sartorii } \\
\text { Xenodon rabdocephalus } \\
\text { Basiliscus vittatus } \\
\text { Corytophanes cristatus } \\
\text { Corytophanes hernandezii } \\
\text { Laemanctus longipes } \\
\text { Laemanctus serratus }\end{array}$ & $\begin{array}{l}\text { Eublepharidae } \\
\text { Geckonidae } \\
\text { Iguanidae } \\
\text { Kinosternidae } \\
\text { Phrynosomatidae } \\
\text { Polychrotidae }\end{array}$ & $\begin{array}{l}\text { Crocodylus moreletii } \\
\text { Micrurus diastema } \\
\text { Rhinoclemmys areolata } \\
\text { Terrapene yucatana } \\
\text { Trachemys venusta } \\
\text { Coleonyx elegans } \\
\text { Hemidactylus frenatus } \\
\text { Sphaerodactylus glaucus } \\
\text { Thecadactylus rapicauda } \\
\text { Ctenosaura alfredschmidti } \\
\text { Claudius angustatus } \\
\text { Kinosternon scorpioides } \\
\text { Sceloporus chrysostictus } \\
\text { Sceloporus lundelli } \\
\text { Anolis biporcatus } \\
\text { Anolis lemurinus } \\
\text { Anolis pentaprion } \\
\text { Anolis rodriguezii } \\
\text { Anolis sericeus } \\
\text { Anolis tropidonotus } \\
\text { Mabuya unimarginata } \\
\text { Scinella cherriei } \\
\text { Ameiva undulata } \\
\text { Aspidocelis angusticeps } \\
\text { Typhlops microstomus } \\
\text { Bothrops asper } \\
\text { Crotalus simus }\end{array}$ \\
\hline
\end{tabular}


Calderón Mandujano et al.: Utilización de hábitat por reptiles en selvas tropicales

\section{LITERATURA CITADA}

Andrews, A. 1990. Fragmentation of habitat by roads and utility corridors: a review. Australian Zoologist 26: 130-42.

Baldwin, E.A., M.N. Marchand \& J.A. Litvaitis. 2004. Terrestrial habitat use by nesting painted turtles in landscapes with different levels of fragmentation. Northeastern Naturalist 11: 41-48.

Begon M., C. R. Townsend \& J. L. Harper. 2006. Ecology: from individuals to ecosystems. 4th Ed. Blackwell Publishing. Malden, MA

Brown, K.S. Jr. 1991. Conservation of Neotropical environments: Insects as indicators. Pp. 350-403 In: N.M. Collins \& J.A. Thomas (Eds). The conservation of insects and their habitats. Fifteenth Symposium of the Royal Entomological Society of London. Academic Press, London.

Bauerle, B., D.L. Spencer \& W. Wheeler. 1975. The use of snakes as a pollution indicator species. Copeia 1975(2): 366-368.

Bierregaard Jr., R.0., T.E. Lovejoy, V. Kapos, A. A. Dos Santos \& R. W. Hutchings. 1992. The biological dynamics of tropical rain forest fragments. A prospective comparison of fragments and continuos forest. BioScience 42: 59-866.

Calderón-Mandujano, R., H. Bahena-Basave, \& S. Calmé. 2005. Guía de anfibios y reptiles de la Reserva de la Biosfera Sian Ka'an y zonas aledañas. Grupo Editorial Keer. Quintana Roo, México

Casas-Andreu G., F. Méndez-De la Cruz \& J. L. Camarillo. 1996. Anfibios y reptiles de Oaxaca. Lista, distribución y conservación. Acta Zoológica Mexicana (n.s.) 69: 1-35.

Campbell, J.A. 1998. Amphibians and reptiles of northern Guatemala, the Yucatán and Belize. Univ. Oklahoma Press, Norman.

Connel, J. H. 1978 Diversity in tropical rain forest and coral reefs. Science 199: 1320-1309

Duellman, W.E. 1963. Amphibians and reptiles of the rainforest of southern El Peten, Guatemala. University of Kansas Publications of the Museum of Natural History 15: 205249.

Gorresen, P. \& M.R. Willing. 2004. Landscape responses of bats to habitat fragmentation in Atlantic forest of Paraguay. Journal of Mammalogy 85: 688-697.

Hanski, I. 1994. A practical model of metapopulation dynamics. Journal of Animal Ecology 63: $151-162$.

Hager, H.A. 1998. Area-sensitivity of reptiles and amphibians: are there indicator species for habitat fragmentation? Ecoscience 5: 139-147.

Herrando-Pérez, S. 2002. Manual de ecología matemática: Un enfoque práctico al análisis multivariado (PCA, Cluster y MDS) para detectar patrones en ecología. Manual de referencia, $2^{a}$ Edición. ECOSUR-Chetumal. Quintana Roo, México

Heyer, R., A. Donnelly, R.W. McDiarmid, L.A.C. Hayek \& M.S. Foster (Eds). 1994. Measuring and monitoring biological diversity: Standard methods for amphibians. Smithsonian Institution Press, Washington, DC.

Hirth, H.F. 1963. The ecology of two lizards on a tropical beach. Ecological Monographs 33: 83-112. 
Hofer, U., L.F. Bersier \& D. Borcard. 2000. Ecotones and gradients as determinants of herpetofaunal community structure in the primary forest of Mount Kupe, Cameroon. Journal of Tropical Ecology 16: 517-533.

INEGI. 1990. Inventario forestal de México. Escala 1: 1,000,000. Instituto Nacional de Geografía y Estadística. México

Islebe, G. 1998. Vegetación de Quintana Roo; vegetación secundaria. Pp. 342-343 In: XacurMaiza, J.A. (Ed). Enciclopedia de Quintana Roo. México.

Kremen, C. 1992. Assessing the indicator properties of species assemblages for natural areas monitoring. Ecological Applications 2: 203-217.

Landres, P.B., J. Verner, \& J.W. Thomas. 1988. Ecological uses of vertebrate indicator species: A critique. Conservation Biology 2: 316-330.

Lee, J.C. 1980. An ecogeographic analysis of the herpetofauna of the Yucatán. University of Kansas Museum of Natural History Miscellaneous Publications 67: 1-75.

Lee, J.C. 1996. The amphibians and reptiles of the Yucatán Peninsula. Comstock Assoc. Ithaca and London.

Lips, K.R., J.K. Reaser, B.E. Young \& R. Ibañez. 2001. El monitoreo de anfibios en América Latina: Un manual para coordinar esfuerzos. SSAR Herpetological Circular No. 30, Ithaca, New York.

Martínez, E., \& C. Galindo-Leal. 2002. La vegetación de Calakmul, Campeche, México: clasificación, composición y distribución. Boletín de la Sociedad Botánica de México 71: 7-32.

Miranda, F. \& E. Hernández. 1963. Los tipos de vegetación de México y su clasificación. Boletín de la Sociedad Botánica de México 28: 29-178

Muñoz, A., R. Martínez-Castellano \& P. Hernández-Martínez. 1996. Anfibios y reptiles de la Reserva El Ocote. Pp. 87-147. In: M. A. Vásquez-Sanchez \& I. March-Mifsut (Eds). Conservación y desarrollo sustentable en la Reserva El Ocote, Chiapas. ECOSURECOSFERA A. C.-CONABIO. México.

Noss, R.F. 1990. Indicators for monitoring biodiversity: a hierarchical approach. Conservation Biology 4: 355-364.

Rzedowski, J. 1983. Vegetación de México. LIMUSA. México.

Roughgarden, J. \& S. Pacala. 1989. Taxon cycle among lizard populations: Review of evidence. Pp. 403-432. In: D. Otte \& J. A. Endler (Eds). Speciation and its consequences. Sinauer Associates. Massachusetts.

Schlaepfer, M.A. \& T.A. Gavin. 2001. Edge effects on lizards and frogs in tropical forest fragments. Conservation Biology 15: 1079-1090.

Schelapfer, M.A., C. Hoober \& C.K. Dodd Jr. 2005. Challenges in evaluating the impact of the trade in amphibians and reptiles on wild populations. BioScience 54: 256-264.

Sousa, W.P. 1984. The role of disturbance in natural communities. Annual Review of Ecology and Systematics 15: 353-91.

Sparrow, H.R., T.D. Sisk, P.R. Ehrlich \& D.D. Murphy. 1994. Techniques and guidelines for monitoring Neotropical butterflies. Conservation Biology 8: 800-809. 
Stork, N.E. 1994. Inventories of biodiversity: more than a question of numbers. Pp. 81-100. In: P.L. Forey, C.J. Humphries \& R.I. Vane-Wright (Eds). Systematics and conservation evaluation. Systematic Association, Special Volume 50. Clarendon Press, Oxford

Smith, G.R. \& R.E. Ballinger. 2001. The ecological consequences of habitat and microhabitat use in lizards: a review. ContemporaryHerpetology Vol. 2001, No. 3. Consultado en línea en: research.calacademy.org/herpetology/herpdocs/ch/2001/3/index.htm.

Tuberville, T.D., J.D. Willson, M.E. Dorcas \& J.W. Gibbons. 2005. Herpetofaunal species richness of southeastern national parks. Southeastern Naturalist 4: 537-569.

Urbina, J.N. \& M.C. Londoño. 2003. Distribución de la comunidad de herpetofauna asociada a cuatro áreas con diferente grado de perturbación en la Isla Gorgona, Pacífico colombiano. Revista de la Academia Colombiana de Ciencias 27: 105-113.

Urbina-Cardona, J.N. \& V.H. Reynoso. 2005. Recambio de anfibios y reptiles en el gradiente potrero-borde-interior en la Reserva de Los Tuxtlas, Veracruz, México. Pp. 191-207. In: G. Halffter, J. Soberón, P. Koleff \& A. Melic (Eds). Sobre Diversidad Biológica: El significado de las diversidades alfa, beta y gamma, Vol. 4. Monografías Tercer Milenio, Zaragoza, España

Urbina-Cardona, J.N., M. Olivares-Pérez \& V.H. Reynoso. 2006. Herpetofauna diversity and microenvironment correlates across a pasture-edge-interior ecotone in tropical rainforest fragments in the Los Tuxtlas Biosphere Reserve of Veracruz, Mexico. Biological Conservation 132: 61-75.

Van Horne, B. 1983. Density as a misleading indicator of habitat quality. Journal of Wildlife Management 47: 893-901.

Recibido: 12 de diciembre de 2006

Aceptado: 19 de octubre de 2007 